\title{
BARREIRAS, ELEMENTOS DIFICULTADORES E FATORES CRÍTICOS NA IMPLEMENTAÇÃO DA GESTÃO DO CONHECIMENTO: UMA REVISÃO DA LITERATURA
}

9

\author{
Fabrício Burger \\ Mestrando em Engenharia e Gestão do Conhecimento pela \\ Universidade Federal de Santa Catarina, Brasil. \\ E-mail: fabriciohere@gmail.com \\ Rodrigo Kraemer \\ Mestre em Engenharia e Gestão do Conhecimento pela \\ Universidade Federal de Santa Catarina, Brasil. \\ E-mail: rodrigokop@gmail.com
}

\section{Gertrudes Aparecida Dandolini}

Doutora em Engenharia de Produção pela Universidade Federal de Santa Catarina, Brasil. Professora da Universidade Federal de Santa Catarina, Brasil.

E-mail: gtude@egc.ufsc.br

\section{João Artur de Souza}

Doutor em Engenharia de Produção pela Universidade Federal de Santa Catarina, Brasil. Professor da Universidade Federal de Santa Catarina, Brasil.

E-mail: jartur@gmail.com

\section{Patrícia de Sá Freire}

Doutora em Engenharia e Gestão do Conhecimento pela Universidade Federal de Santa Catarina, Brasil. Professora da Universidade Federal de Santa Catarina, Brasil. E-mail: patriciadesafreire@gmail.com

\begin{abstract}
Resumo
O ativo conhecimento possui um papel cada vez mais importante no contexto organizacional e sua gestão deve ser realizada para agregar valor. A implementação de Gestão do Conhecimento - GC envolve a introdução de novas tecnologias de informação, mudanças nos processos de negócios, e mudanças na cultura organizacional. Muitos fatores afetam a implementação de GC dentro das organizações. Esses fatores podem ser incentivos ou barreiras para o sistema, e eles podem afetar a eficácia, a eficiência e o desempenho geral do sistema de GC. O objetivo deste artigo é identificar as barreiras ao processo de implementação de GC. Entende-se por barreiras, de acordo com a análise da literatura, os elementos, fatores críticos e os dificultadores que comprometem ou impedem a implementação de GC. Para tanto, uma revisão sistemática da literatura foi realizada com intuito de localizar as publicações que abordem o tema. A análise bibliométrica e descritiva dos resultados apontaram que a ciência tem tratado o tema por três abordagens distintas: identificação das barreiras, hierarquização das barreiras de acordo com sua importância ou discussão sobre como elas afetam a implementação de GC. Além da identificação das publicações que tratam o tema, a principal contribuição deste trabalho, é a identificação das barreiras que deverão ser gerenciadas pelas organizações que pretendem implantar a gestão do conhecimento.
\end{abstract}

Palavras-chave: Implementação. Barreiras. Gestão do Conhecimento. Revisão Sistemática de Literatura.

Perspectivas em Gestão \& Conhecimento, João Pessoa, v. 8, n. 2, p. 43-61, mai./ago. 2018. DOI: http://dx.doi.org/10.21714/2236-417X2018v8n2p43

http://periodicos.ufpb.br/ojs2/index.php/pgc. ISSN: 2236-417X. Publicação sob Licença (cc) EY-NC-ND 


\title{
BARRIERS, DIFFICULTY ELEMENTS AND CRITICAL FACTORS IN THE IMPLEMENTATION OF KNOWLEDGE MANAGEMENT: A REVIEW
}

\begin{abstract}
The active knowledge has an increasingly important role in the organizational context and its management must be carried out to add value. The implementation of Knowledge Management - GC involves the introduction of new information technologies, changes in business processes, and changes in organizational culture. Many factors affect the implementation of KM within organizations. These factors can be incentives or barriers to the system, and they can affect the effectiveness, efficiency, and overall performance of the KM system. The aim of this article is to identify the barriers to the process of $K M$ implementation. Barriers are understood, according to the literature review, as the elements, critical factors and obstacles that compromise or hinder the KM implementation. For this purpose, a systematic review of the literature was carried out in order to locate the publications that approach the theme. The bibliometric and descriptive analysis of the results pointed out that science has dealt with the theme through three different approaches: identification of barriers, hierarchy of barriers according to their importance or discussion of how they affect the KM implementation. In addition to the identification of the publications that deal with the theme, the main contribution of this work is the identification of the barriers that should be managed by the organizations that intend to implement knowledge management.
\end{abstract}

Keywords: Implementation. Barriers. Knowledge management. Systematic Review of Literature.

\section{INTRODUÇÃO}

A partir da década de 80 o conhecimento começou a ser visto com um papel cada vez mais importante para o ambiente competitivo, demandando cada vez mais produtos e serviços de qualidade, diversidade e personalização (WIIG, 1997). Pela visão histórica, percebe-se que a gestão estratégica corporativa tem mudado ao longo do tempo. Anteriormente as organizações buscavam a otimização dos recursos, ou seja, fazer mais com menos recursos. Depois passou para a fabricação de produtos inteligentes, buscando a liderança do produto. Finalmente as organizações se concentraram na criação de soluções buscando uma intimidade com o cliente (WIIG, 1997).

Atualmente, os recursos econômicos fundamentais não são mais o capital, nem os recursos naturais ou a mão de obra, mas sim o conhecimento advindo do capital intelectual. Em todos os setores, as empresas bem-sucedidas são as que têm as melhores informações ou utilizam de forma mais eficaz o seu conhecimento (MACEDO et al., 2010). Para que esse conhecimento possa ser bem articulado, utilizado e mantido na organização, uma gestão adequada do conhecimento precisa ser efetuada. A Gestão do Conhecimento (GC) procura tornar o conhecimento e as experiências mais facilmente disponíveis, para aumentar a eficiência e eficácia da organização (SEDIGHI et al., 2015).

Diante desses desafios teóricos e práticos da gestão contemporânea, a literatura científica passa a sugerir modelos de implementação da GC de modo a nortear as organizações interessadas em atravessar essa fronteira. Por envolver definição de estratégias, capacitação de líderes, reestruturação de características culturais, além de tecnologias, modelagem de processos e envolvimento de pessoas, a implementação de GC exige não só um investimento financeiro substancial, mas também um esforço social e estrutural da organização (WANG; CHANG, 2007). É importante que seja dada uma devida consideração a todos os aspectos da implementação para que essa não resulte em fracasso (ANAND et al., 2015).

A implementação de $\mathrm{GC}$ precisa ter eficácia, pois após o primeiro boom de implementação da GC nas empresas, em muitas os resultados não foram tão satisfatórios

Perspectivas em Gestão \& Conhecimento, João Pessoa, v. 8, n. 2, p. 43-61, mai./ago. 2018. 
(GOLD; MALHOTRA; SEGARS, 2002). Dalkir (2005) afirma que os principais problemas enfrentados resultam de pouco foco nas pessoas e nas questões culturais, como por exemplo, a ênfase na tecnologia, a realização de GC sem o foco no negócio, ou optar pela quantidade de conteúdo em detrimento da qualidade. Assim, Dalkir (2005, p. 304) sugere que a GC deve ser planejada com as decisões de quem (pessoas), o que (conhecimento) e por quê (processos de negócio), sendo que o como (tecnologia) deve ficar para a última decisão. Essa afirmação de Dalkir (2005) vai ao encontro da perspectiva de Servin (2005), que intitulou esses elementos pessoas, processos e tecnologia - como tríade da GC.

Um quarto elemento é sugerido pela Asian Productivity Organization (NAIR; PRAKASH, 2009), a liderança, e a junção destes quatro elementos, pessoas, processo, tecnologia e liderança oferecem à gestão do conhecimento uma nova perspectiva de angariar, nos seus esforços, mais uma importante possibilidade de obter os resultados almejados pela organização (GOMES JR, 2013).

O Comitê Europeu de Normalização (CEN), ampliou as discussões sobre implementação da GC quando desenvolveu o European Guide to Good Practice in Knowledge Management (CEN, 2004), um guia que promoveu o entendimento comum na União Europeia, e atualmente, no mundo. Esse auxilia as organizações, principalmente as micro, pequenas e médias empresas, a implementarem a GC.

Na esfera mundial, a implementação de GC envolve a introdução de novas tecnologias de informação, mudanças nos processos de negócios, e mudanças na cultura organizacional (OLIVEIRA; CALDEIRA; ROMÃO, 2012). Muitos fatores afetam a concepção, implementação e uso de sistemas de GC dentro das organizações. Esses fatores podem ser incentivos ou barreiras para os sistemas, e eles podem afetar a eficácia dos sistemas, a eficiência e o desempenho geral dos sistemas de GC (AHMAD; AN, 2008).

Entender as barreiras na implementação de GC é a base para a construção de mecanismos de superação antes mesmo da implementação em si da GC (DU PLESSIS, 2008), o que, com certeza, como em qualquer projeto, eliminar barreiras significa melhor fluidez do processo, mais otimização de esforços e maior efetividade dos resultados. Entende-se por barreiras (DU PLESSIS, 2008; MILOSZ; MILOSZ, 2010), os elementos (NOURI et al., 2013; WU, 2012; SHANG, 2013), os fatores críticos (SEDIGHI et al., 2015; VALMOHAMMADI, 2010) e os dificultadores (WANG; CHANG, 2007) que possam comprometer ou impedir a implementação de GC.

Neste artigo tem-se como objetivo identificar as barreiras ao processo de implementação de GC, por meio de uma revisão sistemática da literatura em uma base de dados eletrônica, internacional e interdisciplinar.

Este artigo foi estruturado em seis seções, incluindo esta introdução e as referências que apoiaram as argumentações teóricas à pesquisa. A próxima seção descreve os procedimentos metodológicos seguidos. A terceira seção apresenta os resultados da análise bibliométrica e a categorização dos artigos: artigos com foco na identificação de barreiras; artigos com foco na hierarquização das barreiras e artigos que fazem uma discussão sobre as barreiras. A seção quatro traz as análises realizadas dos artigos em cada categoria. A quinta seção traz uma discussão dos resultados. Por fim, na sexta seção, são realizadas as considerações finais.

\section{PROCEDIMENTOS METODOLÓGICOS}

Para a produção desse artigo foi realizada uma revisão sistemática da literatura sobre elementos que dificultam ou impedem a implementação de GC. O método de revisão sistemática de literatura pode ser explicado como uma síntese de estudos primários com

Perspectivas em Gestão \& Conhecimento, João Pessoa, v. 8, n. 2, p. 43-61, mai./ago. 2018. 
objetivos e métodos bem explicitados, realizados através de uma metodologia clara e reprodutível (GREENHALGH, 1997).

Uma busca sistemática foi realizada na base de dados SCOPUS. Ela foi escolhida porque é a maior base de dados de resumos e citações de literatura científica revisada por pares, e oferece a visão mais abrangente sobre a produção de pesquisa do mundo em diversas áreas, considerando como fator relevante a interdisciplinaridade (ELSEVIER, 2016).

A etapa seguinte consistiu na escolha das palavras-chave e período considerado na pesquisa. Visando a total cobertura das publicações sobre o tema, não foi realizado corte temporal e utilizou-se a regra de exaustividade de Bardin (2011). O foco da pesquisa não foi apenas barreiras na implementação da GC, mas todos os elementos dificultadores e fatores críticos que impactam na implementação da GC. Assim, foi definido selecionar primeiramente os trabalhos que discutem a implementação de GC para, depois, selecionar aqueles que tratam desses elementos e fatores que dificultam a implementação. Foram utilizados como palavraschave os termos na seguinte ordem: "knowledge management implementation" OR "implementation of knowledge management" OR "knowledge management deployment". A busca realizada com essas palavras-chave ocorreu em: título (title), resumo (abstract), palavras-chave (keywords). Foram selecionadas apenas as publicações em formato de artigos e reviews, pois entende-se que os mesmos sofreram avaliação por pares. Ao final desta etapa, foram localizadas 206 publicações.

A próxima etapa consistiu na análise dos artigos. Para a seleção, realizou-se a leitura do título, resumo e palavras-chave de cada uma das 206 publicações, aplicando o critério de exclusão dos artigos que não tratavam diretamente do tema em estudo, ou seja, não discutiam barreiras, dificultadores ou fatores críticos na implementação. Após a leitura foram selecionados 26 artigos que tratavam do tema estudado, na perspectiva proposta.

A próxima etapa foi a leitura dos 26 artigos na íntegra. Dos 26 artigos selecionados na terceira etapa, foram excluídos outros cinco artigos, totalizando 21 publicações que se configuraram na amostra deste estudo. Para realizar a análise, dois dos pesquisadores apreciaram separadamente cada artigo a partir da leitura completa, utilizando-se uma das técnicas de codificação de Saldaña (2009). O procedimento em dupla de forma separada foi realizado para diluir o viés da categorização realizada por apenas uma pessoa. Após consenso entre os pesquisadores, as categorias foram definidas e encontram-se explicitadas na próxima seção.

\section{RESULTADOS DA PESQUISA}

Nesta seção, são expostos os resultados da pesquisa. Primeiramente são dispostos alguns dados bibliométricos e, em seguida, é apresentada a categorização que eclodiu na análise com seus respectivos grupos de artigos. Das publicações selecionadas e consideradas neste artigo, o período observado compreendeu os anos entre 2005 e 2015 . Nesse período se destacam três pesquisas publicadas em 2008 e cinco publicadas em 2012, conforme é possível observar no Gráfico 1. 
Gráfico 1 - Ano e número de artigos analisados

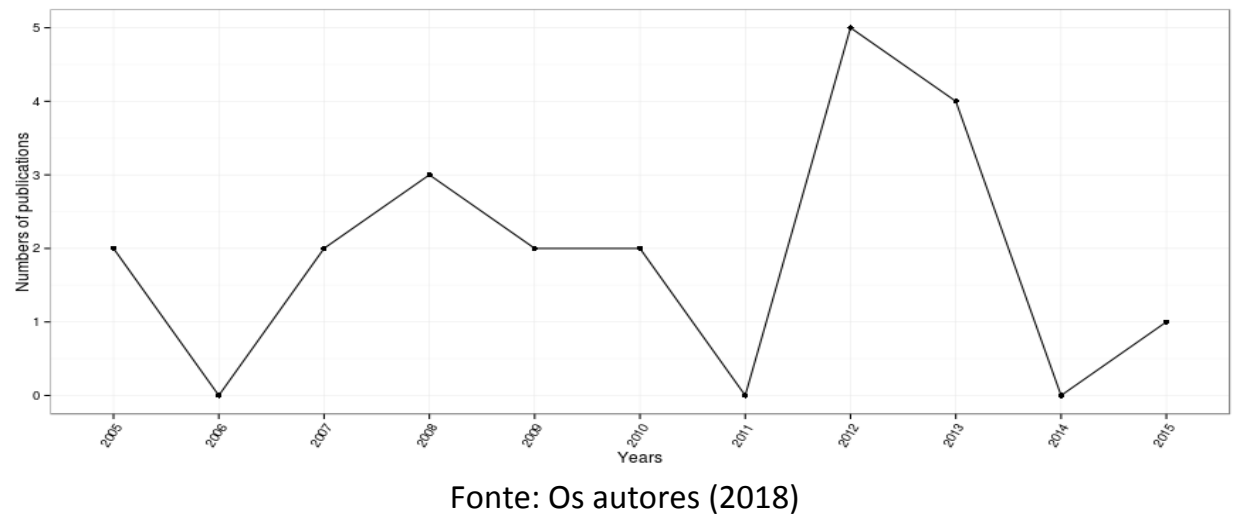

As publicações mais citadas são Lin e Tseng (2005), Wu (2012), Finestone e Snyman (2005) e Wang e Chang (2007) que representam 64\% das citações. A Tabela 1 relaciona as publicações que tiveram ao menos uma citação.

Os artigos foram categorizados com base na forma que as barreiras são abordadas. Foram construídas três categorias de artigos, com as duas primeiras como subcategorias, conforme mostra o Quadro 1.

As categorias e suas subdivisões construídas apresentam as seguintes características:

a) Identificação de barreiras que dificultam a implementação: nessa categoria estão presentes artigos que identificam barreiras, fatores críticos de sucesso ou outros elementos que podem dificultar a implementação bem-sucedida de GC. Há artigos que identificam de maneira geral (mesmos elementos para qualquer implementação), que identificam de maneira específica em um país, setor ou tipo de organização e artigos que identificam os elementos presentes em organizações específicas.

b) Classificação de barreiras que dificultam a implementação: nessa categoria estão presentes artigos que hierarquizam ou classificam os elementos mais importantes que podem dificultar a implementação bem-sucedida de GC, também existindo aqueles que hierarquizam de maneira geral, de maneira específica em um país, setor ou tipo de organização e que criam métodos para hierarquizar em organizações específicas ou fazem a hierarquização em organizações específicas.

c) Discussão sobre as barreiras que dificultam a implementação: aqui foram categorizados três artigos, um que analisa o multiculturalismo, um que classifica em fatores mostrando seus efeitos e possíveis soluções e um artigo que realiza uma previsão se a GC será implementada com sucesso de acordo com a presença de alguns elementos na organização.

Essas categorias são detalhadas e apresentadas após a Tabela 1 e o Quadro 1 mencionados:

Perspectivas em Gestão \& Conhecimento, João Pessoa, v. 8, n. 2, p. 43-61, mai./ago. 2018. 
Tabela 1 - Publicações com citações

\begin{tabular}{|c|c|c|c|}
\hline Autor (es) & Ano & Título & Citações \\
\hline Lin e Tseng & 2005 & $\begin{array}{l}\text { The implementation gaps for the knowledge management } \\
\text { system }\end{array}$ & 60 \\
\hline Wu & 2012 & $\begin{array}{l}\text { Segmenting critical factors for successful knowledge } \\
\text { management implementation using the fuzzy DEMATEL method }\end{array}$ & 37 \\
\hline $\begin{array}{l}\text { Finestone e } \\
\text { Snyman }\end{array}$ & 2005 & $\begin{array}{l}\text { Corporate South Africa: Making multicultural knowledge sharing } \\
\text { work }\end{array}$ & 26 \\
\hline Wang e Chang & 2007 & $\begin{array}{l}\text { Application of consistent fuzzy preference relations in predicting } \\
\text { the success of knowledge management implementation }\end{array}$ & 25 \\
\hline Du Plessis & 2008 & $\begin{array}{l}\text { What bars organizations from managing knowledge } \\
\text { successfully? }\end{array}$ & 19 \\
\hline Kant e Singh & 2009 & $\begin{array}{l}\text { Knowledge management implementation: Modelling the } \\
\text { variables }\end{array}$ & 13 \\
\hline $\begin{array}{l}\text { Wickramasinghe, } \\
\text { Bali e Geisler }\end{array}$ & 2007 & $\begin{array}{l}\text { The major barriers and facilitators for the adoption and } \\
\text { implementation of knowledge management in healthcare } \\
\text { operations }\end{array}$ & 9 \\
\hline Kant e Singh & 2008 & $\begin{array}{l}\text { Knowledge Management Implementation: Modeling the } \\
\text { Barriers }\end{array}$ & 8 \\
\hline Milosz e Milosz & 2010 & $\begin{array}{l}\text { Critical success factors and barriers to implementation of } \\
\text { knowledge management systems at Polish SMEs }\end{array}$ & 7 \\
\hline Siddique & 2012 & $\begin{array}{l}\text { Knowledge management initiatives in the United Arab Emirates: } \\
\text { A baseline study }\end{array}$ & 7 \\
\hline Ahmad e An & 2008 & $\begin{array}{l}\text { Knowledge management implementation in construction } \\
\text { projects: a KM model for Knowledge Creation, Collection and } \\
\text { Updating (KCCU) }\end{array}$ & 6 \\
\hline $\begin{array}{l}\text { Picker, Ruhnke e } \\
\text { Leker }\end{array}$ & 2009 & Developing knowledge management -what makes the success? & 5 \\
\hline Valmohammadi & 2010 & $\begin{array}{l}\text { Investigation and assessment of critical success factors of } \\
\text { knowledge management implementation in Iranian small-to- } \\
\text { medium sized enterprises }\end{array}$ & 3 \\
\hline $\begin{array}{l}\text { Oliveira, Caldeira } \\
\text { e Romão }\end{array}$ & 2012 & $\begin{array}{l}\text { Knowledge Management Implementation: An Evolutionary } \\
\text { Process in Organizations }\end{array}$ & 3 \\
\hline $\begin{array}{l}\text { Anand, Kant e } \\
\text { Singh }\end{array}$ & 2013 & Knowledge sharing in SMEs: Modelling the barriers & 3 \\
\hline $\begin{array}{l}\text { Khalifa e } \\
\text { Jamaluddin }\end{array}$ & 2012 & $\begin{array}{l}\text { Key success factors affecting knowledge management } \\
\text { implementation in construction industry in Libya }\end{array}$ & 1 \\
\hline
\end{tabular}

Fonte: Os autores (2018)

Quadro 1 - Categorias e subcategorias dos artigos

\begin{tabular}{|c|c|l|}
\hline Categorias & \multicolumn{1}{|c|}{ Subcategorias } & \multicolumn{1}{c|}{ Artigos } \\
\hline \multirow{2}{*}{ Identificação } & Geral & $\begin{array}{l}\text { (LIN; TSENG, 2005); (DU } \\
\text { PLESSIS, 2008); (PICKER; } \\
\text { RUHNKE; LEKER, 2009) }\end{array}$ \\
& & $\begin{array}{l}\text { (VALMOHAMMADI, 2010); } \\
\text { (MILOSZ; MILOSZ, 2010); } \\
\text { (SIDDIQUE, 2012); }\end{array}$ \\
\cline { 2 - 3 } & País, tipo ou setor específico & \\
& &
\end{tabular}

Perspectivas em Gestão \& Conhecimento, João Pessoa, v. 8, n. 2, p. 43-61, mai./ago. 2018. 


\begin{tabular}{|c|c|c|}
\hline & & $\begin{array}{l}\text { (WICKRAMASINGHE et al., } \\
\text { 2007); (KHALIFA; JAMALUDDIN, } \\
\text { 2012) }\end{array}$ \\
\hline & Organização específica & $\begin{array}{l}\text { (MIRPOOR et al.., 2013); (SHOO } \\
\text { et al., 2012) }\end{array}$ \\
\hline \multirow[t]{3}{*}{ Classificação } & Geral & (KANT; SINGH, 2008; 2009) \\
\hline & País, tipo ou setor específico & $\begin{array}{l}\text { (SEDIGHI et al.., 2015); (ANAND } \\
\text { et al.., 2013) }\end{array}$ \\
\hline & Organização específica & $\begin{array}{l}\text { (NOURI et al., 2013); (WU, } \\
\text { 2012); (SHANG, 2013); } \\
\text { (OLIVEIRA et al., 2012) }\end{array}$ \\
\hline \multicolumn{2}{|c|}{ Discussão } & $\begin{array}{l}\text { (FINESTONE; SNYMAN, 2005); } \\
\text { (AHMAD; AN, 2008); (WANG; } \\
\text { CHANG, 2007) }\end{array}$ \\
\hline
\end{tabular}

Fonte: Os autores (2018)

\subsection{Identificação das barreiras}

Os elementos que possam se constituir de obstáculos, barreiras ou dificultadores na implementação da GC podem ser identificados de maneira geral, podendo servir para qualquer implementação de GC.

Para evitar dificuldades na implementação de GC, Lin e Tseng (2005) constroem um framework genérico cujo objetivo é mostrar possíveis lacunas na implementação de sistemas de GC. Realizando proposições teóricas, os autores identificam seis lacunas que podem ocorrer. A primeira delas é a diferença entre o conhecimento necessário para aumentar a competitividade de uma empresa como percebido pela alta gestão e o conhecimento realmente necessário para melhorar a sua competitividade. A segunda é a lacuna entre o conhecimento necessário para aumentar a competitividade de uma empresa como percebido pela alta gestão e o plano para implementar a gestão do conhecimento. A terceira se refere à diferença entre o plano para implementar a gestão do conhecimento como proposto pela alta gestão e o progresso da implementação do plano de gestão do conhecimento. A quarta, está configurada pela diferença entre o conhecimento obtido após a implementação do sistema de gestão do conhecimento e os conhecimentos necessários para aumentar a competitividade de uma empresa. Já a quinta é a lacuna entre o conhecimento necessário para aumentar a competitividade de uma empresa, como percebido pela alta gestão e por outros funcionários. Por fim, a sexta, se refere à lacuna entre o conhecimento necessário para aumentar a competitividade de uma empresa como percebido pelos funcionários e o conhecimento efetivamente obtido após a implementação do sistema de gestão do conhecimento. 0 framework serve de referência às organizações para a implementação de sistemas de GC. Constata-se, neste estudo, a falta de alinhamento entre teoria e prática; entre o que é necessário e deve ser feito e o que de fato é realizado no contexto organizacional.

Procurando dar uma visão geral das potenciais barreiras na implementação de GC, Du Plessis (2008) faz uma listagem das seguintes barreiras: definição de conhecimento e de GC, confidencialidade do conhecimento, baixo aproveitamento de sistemas de GC devido à pouca compreensão da GC, linguagem, cultura organizacional, entendimento comum de GC, aceitação da GC, baixas taxas de retenção de pessoal, altos custos de implementação em comparação com valor agregado, compreensão das necessidades e razões para a GC, tecnologia (uso, custo, manutenção), habilidades diferentes entre os membros da equipe, estilo de gestão, aceitação do usuário, consciência dos conhecimentos críticos da organização. $\mathrm{O}$ autor se mostra mais pragmático nas suas considerações na medida que seus resultados

Perspectivas em Gestão \& Conhecimento, João Pessoa, v. 8, n. 2, p. 43-61, mai./ago. 2018. 
apontam barreiras de diversas naturezas e alinhadas com os resultados de Lin e Tseng, por exemplo, quando cita a compreensão das necessidades e razões para a GC.

Além dos elementos gerais identificados por Du Pleassis (2008), podem existir elementos que são específicos para determinados tipos de empresa, um determinado setor ou para um país em específico. Em relação às pequenas e médias empresas (PMEs), Milosz e Milosz (2010) aplicaram questionários em empresas na Polônia e relataram as principais barreiras em relação à implementação de GC, que seriam a codificação de conhecimento, o armazenamento de conhecimento, a propriedade intelectual e a estrutura organizacional. Outro achado é que $76 \%$ das PMEs não possuem a GC em sua estratégia.

Ainda em PMEs, Valmohammadi (2010) procura identificar fatores críticos de sucesso para a implementação de GC nessas organizações do Irã. O autor identificou 12 fatores críticos de sucesso na literatura - liderança, cultura organizacional, sistemas de informação, estratégia de GC, medição de desempenho, infraestrutura organizacional, processo e atividades, recompensa e motivação, treinamento e educação, recursos, gestão de pessoas e benchmarking. Ele avalia os fatores junto a peritos iranianos, donde aponta que $80 \%$ dos peritos consideram esses fatores satisfatórios e favorecerão a implementação de GC em PMEs Iranianas.

Procurando gerar alguns dados iniciais sobre iniciativas e práticas de GC nos Emirados Árabes Unidos, Siddique (2012) analisa 270 questionários, para descobrir os benefícios e problemas que as empresas deste país encontraram no processo de implementação da GC. A mais importante barreira para o avanço das práticas de GC nos Emirados Árabes Unidos foi a má compreensão da GC em teoria e prática. Também encontraram que a GC ainda não foi incorporada na estratégia ou declaração de missão na maioria das empresas pesquisadas e falta de cultura organizacional adequada e pouco envolvimento de gestores em programas de GC também foram vistos como as principais barreiras. A maioria das empresas também não fornecem incentivos suficientes para os funcionários.

Analisando as barreiras na implementação de GC em organizações de saúde, Wickramasinghe et al. (2007) identificaram quatro fatores que podem trazer dificuldades: tecnológicos, organizacionais, humanos e econômicos. O fator tecnológico se refere aos atributos inerentes à tecnologia, tais como a compatibilidade com outros sistemas, complexidade e testagem; a aplicabilidade à tarefa para a qual a tecnologia está sendo adotada; a facilidade de manutenção; a qualidade (em termos de erros, avarias, e não à capacidade de resposta); a facilidade de atualização ou substituição. O fator organizacional inclui as tradicionais barreiras para a adoção da tecnologia, tais como rivalidades políticas, falta de apoio da alta administração para tal tecnologia e inovação, e experiência prévia da organização com tipos similares de tecnologias e sua implementação; e eventos passados malsucedidos tendem a impedir quaisquer tentativas atuais de adotar e implementar a tecnologia. $O$ fator humano inclui as barreiras culturais, a curva de aprendizagem necessária para implementar a tecnologia e da percepção desfavorável do papel da tecnologia, o seu valor para a organização, e suas chances de contribuir com sucesso para tarefas e objetivos da organização. O fator econômico inclui os custos da tecnologia, e a relação custo-benefício de implementação de sistemas tecnológicos caros. Os autores focam a GC apenas sob o ponto de vista da implementação de tecnologia, pois mesmo as barreiras humanas são mostradas em como as pessoas usarão a nova tecnologia implementada.

Khalifa e Jamaluddin (2012) procuram identificar os principais fatores de sucesso que afetam a implementação de GC no setor de construção da Líbia. Com base na literatura encontraram fatores-chave os quais foram testados com testes de hipóteses para verificar a correlação com a implementação da GC. Encontraram relações significativas no fator apoio da alta gerência na implementação da GC e no fator compartilhamento de conhecimento. Também encontraram correlação positiva em treinamento e educação, cultura de

Perspectivas em Gestão \& Conhecimento, João Pessoa, v. 8, n. 2, p. 43-61, mai./ago. 2018. 
compartilhamento, facilidade de uso, auditoria de conhecimento e equipe de GC. Não foram encontradas relações significativas em cultura organizacional, motivação em compartilhamento, infraestrutura de TI e estrutura de conhecimento.

Direcionando ainda mais o foco da análise, as barreiras podem ser identificadas em uma organização específica. Mirpoor et al. (2013) procuram identificar os fatores de influência em uma implementação de GC de um escritório de alfândega no Irã. Os autores aplicaram questionários com gestores e funcionários sobre cinco fatores previamente identificados na literatura: cultura organizacional, apoio da alta gestão, preparação dos funcionários para a GC, estrutura organizacional e tecnologia. Os resultados encontrados indicam que três foram considerados mais influentes (cultura, apoio da alta gestão e preparação) e os outros dois menos (estrutura organizacional e tecnologia).

Realizando um estudo na Fundação Médica e de Pesquisa da África - AMREF, Shoo et al. (2012) procuraram analisar a implementação de de GC. Aplicando questionários e realizando grupos focais e entrevistas em profundidade, os problemas e limitações encontrados foram: uma falta de incentivos para documentação e disseminação; documentação limitada e falta de uso de boas práticas de programação; e atenção superficial para resultados ou uso de conhecimento baseado em evidências.

\subsection{Hierarquização e classificação de barreiras}

Além de ser identificadas, as barreiras podem ser também hierarquizadas ou classificadas por ordem de importância.

De uma maneira geral, Kant e Singh (2008) procuram identificar e classificar as barreiras para a implementação de GC. Com base em 19 barreiras encontradas na literatura, os autores as analisaram utilizando uma Modelagem Estrutural Interpretativa (Interpretive Structural Modeling - ISM) que é uma metodologia para identificar relações entre variáveis. As barreiras apresentadas na pesquisa como Knowledge Management Barriers (KMB) foram classificadas em quatro categorias: KMB autônomas, KMB dependentes, KMB de ligação e KMB independentes. Realizando um agrupamento os autores descobriram que não há barreiras autônomas e nem barreiras de ligação. As barreiras dependentes são as de número 11 a 20 e as independentes são de número 1 a 10 e as relações de dependência são mostradas na Figura 1.

Assim, segundo os autores, eliminadas as primeiras barreiras que são independentes, tem-se maior possibilidade de eliminar as demais, dependentes, o que pode nortear os esforços para a obtenção de sucesso no processo.

Há também estudos que direcionam para realidades mais específicas a hierarquização de barreiras, como para setores específicos de um país.

Perspectivas em Gestão \& Conhecimento, João Pessoa, v. 8, n. 2, p. 43-61, mai./ago. 2018. 
Figura 1 - Hierarquia e relação das barreiras na implementação de GC
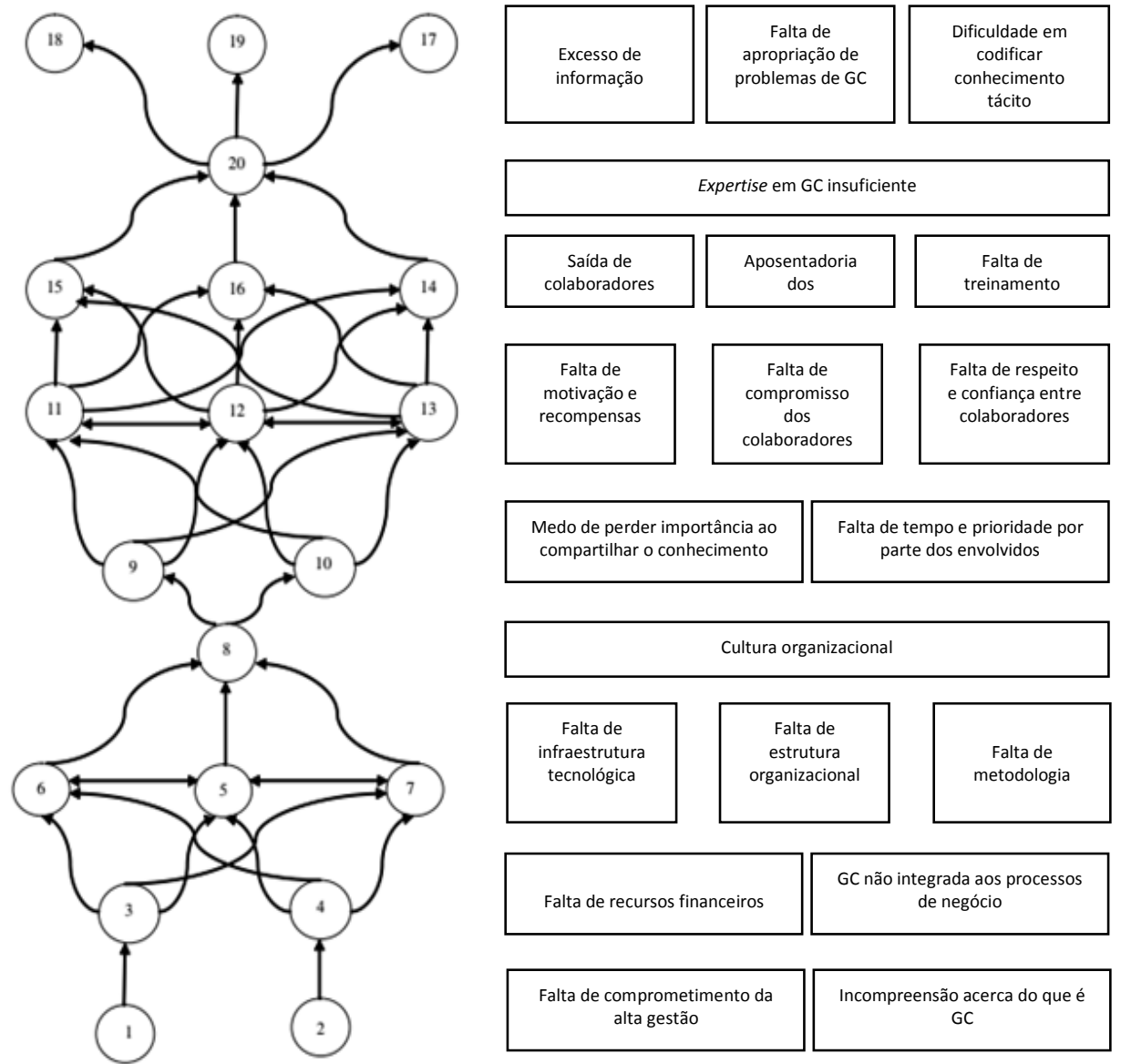

Fonte: Kant e Singh (2008, tradução nossa)

Através do uso da técnica de análise hierárquica de processos (Analytic Hierarchy Process - AHP), Sedighi et al. (2015) procuram identificar e classificar os Fatores Críticos de Sucesso mais importantes para a implementação de GC no setor de energia iraniano. A classificação visa identificar as barreiras A partir de 33 fatores da literatura (Quadro 2) divididos em oito categorias, os autores hierarquizam os mais importantes para essa realidade. As categorias classificadas por ordem de importância são: cultura corporativa, recursos humanos e financeiros, estratégia e liderança, estrutura e procedimentos, fatores intermediários, processos de GC, fatores macro, tecnologia e infraestrutura. Os fatores classificados por importância são os mostrados no Quadro 2.

Quadro 2 - Fatores críticos de sucesso e categorias associadas por ordem de importância

\begin{tabular}{|c|c|c|}
\hline Ranking Global & Fatores & Categorias \\
\hline 1 & Compartilhamento do conhecimento & Cultura Organizacional \\
\hline 2 & Supply Chain & Fatores Intermediários \\
\hline 3 & Cultura colaborativa & Cultura Organizacional \\
\hline 4 & Cultura de socialização & Cultura Organizacional \\
\hline 5 & Gestão da mudança & Estratégia e Liderança \\
\hline
\end{tabular}

Perspectivas em Gestão \& Conhecimento, João Pessoa, v. 8, n. 2, p. 43-61, mai./ago. 2018. 
Fabrício Burger et al.

\begin{tabular}{|c|c|c|}
\hline 6 & Processos de GC & Processos de GC \\
\hline 7 & Investimentos Financeiros & Recursos Humanos e Financeiros \\
\hline 8 & Sistema de incentivos & Estrutura e Procedimentos \\
\hline 9 & Compromisso da alta gestão & Estratégia e Liderança \\
\hline 10 & Concorrentes comerciais & Fatores Intermediários \\
\hline 11 & Ferramenta de mensuração da GC & Processos de GC \\
\hline 12 & Gestão de recursos humanos & Recursos Humanos e Financeiros \\
\hline 13 & Habilidade de trabalho em equipe & Recursos Humanos e Financeiros \\
\hline 14 & Tamanho da organização & Estrutura e Procedimentos \\
\hline 15 & Programa de empoderamento & Recursos Humanos e Financeiros \\
\hline 16 & Sistema de educação & Fatores Macro \\
\hline 17 & Sistema de segurança da informação & Tecnologia e Infraestrutura \\
\hline 18 & Estrutura organizacional & Estrutura e Procedimentos \\
\hline 19 & Propriedade intelectual & Tecnologia e Infraestrutura \\
\hline 20 & Estratégia de GC & Estratégia e Liderança \\
\hline 21 & Fatores econômicos & Fatores Macro \\
\hline 22 & Treinamento de colaboradores & Recursos Humanos e Financeiros \\
\hline 23 & Canais de transferência do conhecimento & Estrutura e Procedimentos \\
\hline 24 & Fatores políticos & Fatores Macro \\
\hline 25 & Acesso à tecnologia de comunicação & Tecnologia e Infraestrutura \\
\hline 26 & Processo de globalização & Fatores Macro \\
\hline 27 & Fatores tecnológicos & Fatores Macro \\
\hline 28 & Usabilidade do sistema & Tecnologia e Infraestrutura \\
\hline 29 & Relações sociais & Fatores Macro \\
\hline 30 & Mecanismos de busca & Tecnologia e Infraestrutura \\
\hline 31 & Rede / Comunidade de prática & Estrutura e Procedimentos \\
\hline 32 & Fatores de legislação & Fatores Macro \\
\hline 33 & Sistema de comunicação & Tecnologia e Infraestrutura \\
\hline
\end{tabular}

Fonte: Sedighi et al. (2015, tradução nossa)

É possível observar que os fatores humanos foram apontados como mais relevantes que os tecnológicos. O estudo de Anand, Kant e Singh (2013) corrobora com essa afirmação. Eles aplicaram um questionário em PMEs com o objetivo de classificar quais barreiras são mais influentes no compartilhamento do conhecimento em organizações desse porte. Os resultados, utilizando também a metodologia de modelagem estrutural interpretativa (Interpretive Structural Modelling - ISM), apontaram a falta de comprometimento da alta direção e a falta de entendimento sobre o que é GC como barreiras relevantes na

Perspectivas em Gestão \& Conhecimento, João Pessoa, v. 8, n. 2, p. 43-61, mai./ago. 2018. 
implementação de GC nas organizações de pequeno e médio porte (ANAND; KANT; SINGH, 2013).

Outros trabalhos realizam hierarquizações em uma determinada organização (NOURI et al., 2013), são criadas metodologias para realizar a hierarquia em organizações (WU, 2012; SHANG, 2013) ou então os elementos são hierarquizados de acordo com o nível ou fase de implementação da GC (OLIVEIRA et al., 2012).

Nouri et al. (2013) procuram ordenar os capacitadores - que podem ser interpretados como fatores críticos ou barreiras - relacionados à GC com base em duas estratégias de implementação de GC ocorridas na Companhia Nacional de Petróleo do Irã. Os autores encontraram a liderança como o principal fator na estratégia de personalização, e a tecnologia da informação na estratégia de codificação como o fator mais importante.

Wu (2012) afirma que é importante observar os fatores críticos de GC em implementação eficaz, mas nem todos são significativos ao mesmo tempo. Como determinar a importância dos fatores críticos envolve a imprecisão da decisão humana, o autor procura segmentar os fatores críticos de sucesso na implementação de GC, utilizando métodos matemáticos como a teoria dos conjuntos fuzzy e teoria dos grafos, e apresenta o modelo DEMATEL. No artigo o autor realiza um estudo de caso em uma determina empresa e mostra ser possível segmentar os fatores críticos, encontrando como resultados de fatores críticos principais, cultura e pessoas.

Shang (2013) afirma que para se implementar com sucesso a GC em organizações, é necessário observar alguns fatores críticos de sucesso. Segundo o autor, a literatura apresenta vários fatores, no entanto, diversas empresas têm características diferentes, diferentes atribuições e os fatores têm pesos diferentes em cada empresa. Assim, ele utiliza o modelo ANOVA que emprega o método de análise de variância para selecionar os fatores mais importantes, utilizando os conjuntos fuzzy e redes neurais, possibilitando uma seleção automatizada para auxiliar a implementar a GC.

A partir de um novo framework, com diferentes estágios, para orientar a implementação de GC nas organizações Oliveira et al. (2012) também analisam os fatores associados à implementação separados por fase. Os fatores foram classificados pelo autor em ordem de importância de acordo com o estágio. São eles: 1a fase - planejamento: apoio da alta gerência, cultura organizacional, estrutura organizacional, alinhamento com os objetivos de negócios, objetivos, orçamento; 2a fase - iniciação: conhecimento explícito, conhecimento do líder do projeto de gestão, fases do processo, tecnologia e tempo; 3 a fase - desenvolvimento: treinamento, sistemas de recompensa, comunicação, conhecimento tácito, benefícios e conhecimento básico; 4a fase - integração: legislação, clientes, fornecedores, parceiros e concorrentes. Assim, as organizações não precisam se preocupar com todos os fatores ao mesmo tempo, mas com os relevantes em cada estágio, que são mantidos nas próximas fases e incorporados com os demais fatores.

\subsection{Discutem sobre as barreiras}

Alguns artigos fazem uma discussão sobre as barreiras, seja analisando uma em especial, o multiculturalismo (FINESTONE; SNYMAN, 2005), seja classificando-os em fatores, mostrando seus efeitos e discutindo possíveis soluções (AHMAD; AN, 2008) ou propõem um modelo de previsão de sucesso na implementação de acordo com a presença de elementos dificultadores (WANG; CHANG, 2007).

Finestone e Snyman (2005) discutem sobre um fator que pode influenciar a implementação de GC que é o multiculturalismo, muito presente na cultura corporativa sulafricana. Realizando entrevistas em profundidade em três grandes empresas, os autores percebem que a presença de várias culturas em um mesmo ambiente corporativo pode ser

Perspectivas em Gestão \& Conhecimento, João Pessoa, v. 8, n. 2, p. 43-61, mai./ago. 2018. 
vista como uma vantagem ou obstáculo. A vantagem mais evidente é a existência de uma diversidade de ideias, influências e culturas, que são fundamentais para a inovação. Com relação às barreiras, o multiculturalismo pode provocar: excesso de diversidade na resolução de problemas que pode ser disfuncional; empresas multiculturais podem achar que é difícil chegar a um único acordo sobre questões podendo levar a discordância sobre ações; a diversidade pode produzir dinâmicas negativas, como o etnocentrismo, estereótipos e choques culturais; ele tem o potencial para polarizar diferentes grupos sociais e danos na produtividade; e a ignorância das diferenças culturais é uma causa de mal-entendidos e, como resultado, conflitos.

Ahmad e An (2008) propõem um novo modelo de implementação de GC baseado em grandes empresas de construção, mas também classificam os fatores que afetam a implementação da GC e discutem sobre como tratá-los. Esses fatores são classificados em cinco categorias e seus efeitos sobre o sistema de GC são ilustrados no Quadro 3:

Quadro 3 - Fatores que afetam a implementação de GC e seus efeitos

\begin{tabular}{|c|c|}
\hline Fatores & Efeitos \\
\hline Individuais & $\begin{array}{l}\text { Cultura pessoal, tais como valores, normas e comportamentos } \\
\text { Nível de confiança entre os funcionários } \\
\text { Motivação, treinamento e suporte } \\
\text { Comprometimento, comunicação e competências } \\
\text { Experiência com sistemas de computadores de TI e } \\
\text { Experiência com pacotes de software e sistemas operacionais } \\
\text { Disponibilidade de conhecimentos de projetos passados e atuais } \\
\text { Métodos de avaliação de desempenho dos funcionários }\end{array}$ \\
\hline Organizacionais & $\begin{array}{l}\text { Apoio à gestão, compromisso e consciência } \\
\text { Estratégia de gestão e visão } \\
\text { A concorrência com outras organizações } \\
\text { Globalização (Organização doméstica ou internacional) } \\
\text { Estrutura organizacional e política } \\
\text { Processos de negócios e operações } \\
\text { Métodos de monitorização e avaliação }\end{array}$ \\
\hline Tecnológicos & $\begin{array}{l}\text { Infraestrutura de TI e sistemas de apoio } \\
\text { Especificações de hardware: velocidade, capacidade e flexibilidade } \\
\text { Especificações de software: a disponibilidade e facilidade de uso de software, } \\
\text { captura de dados e ferramentas de análise e ferramentas de integração de dados. } \\
\text { Disponibilidade e especificação de TIC. } \\
\text { Mudança contínua e avanços na indústria. } \\
\text { Métodos e ferramentas disponíveis para GC }\end{array}$ \\
\hline Econômicos & $\begin{array}{l}\text { Custo de hardware, ambiente de trabalho, acessórios e redes } \\
\text { Custo de aquisição de software, implementação e manutenção } \\
\text { Custos de gestão e operações de conhecimentos } \\
\text { Capacidades financeiras da organização } \\
\text { Nível de rentabilidade dos projetos }\end{array}$ \\
\hline Clientes & $\begin{array}{l}\text { O aumento da demanda de clientes para: } \\
\text { - Menos tempo e custo do projeto e atividade de conclusão } \\
\text { - Melhoria da qualidade dos produtos } \\
\text { - Melhoria da gestão da cadeia de suprimentos } \\
\text { - Melhoria da gestão de relacionamento com clientes }\end{array}$ \\
\hline
\end{tabular}

Perspectivas em Gestão \& Conhecimento, João Pessoa, v. 8, n. 2, p. 43-61, mai./ago. 2018. 


\begin{tabular}{|c|c|}
\hline & - Melhoria da resposta para mudança dos pedidos do cliente \\
\hline Outros & $\begin{array}{l}\text { Questões legais } \\
\text { Segurança da informação e privacidade } \\
\text { Apoio governamental } \\
\text { Segurança e saúde }\end{array}$ \\
\hline
\end{tabular}

Fonte: Ahmad e An (2008, tradução nossa)

De acordo com Ahmad e $\mathrm{Na}$ (2008), os fatores individuais, relacionado a alguns comportamentos e resistência individual pode afetar negativamente os processos de GC e da arquitetura do sistema de GC na organização. Para reduzir os efeitos desses fatores individuais, a gestão tem de incentivar a criação e compartilhamento de conhecimento através de sistemas de recompensa e de avaliação de desempenho organizacional, mostrando compromisso e fornecendo os recursos necessários para implementar e usar o sistema de GC. Em relação ao segundo fator, o apoio da gestão é importante e os gestores devem incentivar e apoiar o uso e implementação de iniciativas de GC, para ajudar a equipe de GC no desenvolvimento e melhoria. As mudanças na tecnologia também afetam os sistemas de GC. Em relação à tecnologia, os sistemas de GC devem ser concebidos e regularmente melhorados para satisfazer tais mudanças e melhoria das tecnologias de construção e de TI. Conteúdos também devem ser regularmente revistos e os conhecimentos desatualizados e inválidos devem ser removidos dos sistemas. Em relação aos aspectos econômicos, a GC também requer o uso e consumo de outros recursos organizacionais, como dinheiro, tempo e esforço, e quanto mais o sistema de GC for financeiramente viável, mais ele poderá inspirar os esforços de GC. O quinto fator, a demanda dos clientes é uma das pressões ambientais importantes em GC. A finalidade principal do sistema de GC é ser flexível o suficiente não só para atender às novas demandas dos clientes, mas também para superar suas expectativas. Outros fatores, como a segurança do conhecimento e privacidade devem fazer um equilíbrio entre abertura e proteção dos seus sistemas de conhecimento. Demasiada abertura pode ameaçar a vantagem competitiva, enquanto muita proteção pode afetar negativamente o processo de inovação e incentivar a burocracia e hierarquia na organização.

Wang e Chang (2007) propõem um modelo de previsão hierárquica analítica com base em relações de preferência fuzzy para as organizações tornarem consciência dos fatores que afetam a GC para uma implementação bem-sucedida. Os autores utilizam sete fatores-chave características do pessoal, projeto de GC, estrutura organizacional, liderança, auditoria e avaliação, cultura organizacional e tecnologia e propõem um modelo para avaliar a porcentagem de sucesso ou fracasso na implementação de GC: se um fator de influência está fortemente presente na organização, a probabilidade de implementação ser bem-sucedida é maior que a probabilidade de fracasso.

\section{DISCUSSÃO}

Ao comparar os estudos científicos encontrados na base de dados Scopus, identifica-se que existem três diferentes abordagens de análise: identificação das barreiras (LIN; TSENG, 2005); (DU PLESSIS, 2008); (PICKER; RUHNKE; LEKER, 2009); (VALMOHAMMADI, 2010); (MILOSZ; MILOSZ, 2010); (SIDDIQUE, 2012); (WICKRAMASINGHE et al., 2007); (KHALIFA; JAMALUDDIN, 2012); (MIRPOOR et al., 2013); (SHOO et al., 2012); classificação por grau de importância (KANT; SINGH, 2008; 2009); (SEDIGHI et al., 2015); (ANAND et al., 2013); (NOURI et al., 2013); (WU, 2012); (SHANG, 2013); (OLIVEIRA et al., 2012), ou discussão sobre as

Perspectivas em Gestão \& Conhecimento, João Pessoa, v. 8, n. 2, p. 43-61, mai./ago. 2018. 
barreiras na implementação da GC (FINESTONE; SNYMAN, 2005); (AHMAD; AN, 2008); (WANG; CHANG, 2007).

Os trabalhos que objetivam identificar as diferentes barreiras a implementação de GC ainda podem ser subdivididos entre três abordagens para a classificação dessas barreiras. Uns apontam que as barreiras podem ser identificadas de maneira geral, com as mesmas barreiras em qualquer implementação (LIN; TSENG, 2005); (DU PLESSIS, 2008); (PICKER; RUHNKE; LEKER, 2009). Outros apontam que as barreiras surgem dependendo de influências específicas de um país, setor ou tipo de organização (VALMOHAMMADI, 2010); (MILOSZ; MILOSZ, 2010); (SIDDIQUE, 2012); (WICKRAMASINGHE et al., 2007); (KHALIFA; JAMALUDDIN, 2012). Por fim, algumas publicações buscam identificar as barreiras presentes em organizações específicas (MIRPOOR ET AL., 2013); (SHOO et al., 2012). Os trabalhos que hierarquizam também podem classificar de maneira geral (KANT; SINGH, 2008; 2009), de maneira específica em um país, setor ou tipo de organização (SEDIGHI et al., 2015); (ANAND et al., 2013), criar métodos para hierarquizar em organizações específicas (WU, 2012; SHANG, 2013), fazer a hierarquização em organizações específicas (NOURI et al., 2013) ou então os elementos são hierarquizados de acordo com o nível ou fase de implementação da GC (OLIVEIRA et al., 2012). Já os que discutem sobre as barreiras que dificultam a implementação tratam do multiculturalismo (FINESTONE; SNYMAN, 2005), classificam em fatores mostrando seus efeitos e possíveis soluções (AHMAD; AN, 2008) ou realizando uma previsão se a GC será implementada com sucesso de acordo com a presença de algumas barreiras na organização (WANG; CHANG, 2007).

Realizando um levantamento das barreiras citadas, gera-se o Quadro 4 que apresenta as barreiras que tiveram mais citações nos trabalhos analisados:

Quadro 4 - Fatores que afetam a implementação de GC e seus efeitos

\begin{tabular}{|c|l|l|}
\hline \multicolumn{2}{|c|}{ Barreiras } & \multicolumn{1}{c|}{ Autores } \\
\hline 1 & $\begin{array}{l}\text { Cultura organizacional não } \\
\text { alinhada }\end{array}$ & $\begin{array}{l}\text { Siddique (2012); Wickramasinghe } \text { et al. (2007); Du Plessis } \\
\text { (2008); Valmohammadi (2010); Mirpoor et al. (2013); } \\
\text { Sedighi } \text { et al. (2015); Oliveira et al. (2012); Wang e Chang } \\
\text { (2007) }\end{array}$ \\
\hline 2 & $\begin{array}{l}\text { Falta de engajamento e apoio da } \\
\text { liderança }\end{array}$ & $\begin{array}{l}\text { Siddique (2012); Wickramasinghe } \text { et al. (2007); Khalifa e } \\
\text { Jamaluddin (2012); Mirpoor et al. (2013); Sedighi } \text { et al. } \\
\text { (2015); Anand, Kant e Singh (2013); Nouri et al. (2013); } \\
\text { Oliveira et al. (2012) }\end{array}$ \\
\hline 3 & $\begin{array}{l}\text { Dificuldades com } \\
\text { sistemas/tecnologia }\end{array}$ & $\begin{array}{l}\text { Wickramasinghe } \text { et al. (2007); Du Plessis (2008); Milosz e } \\
\text { Milosz (2010); Shoo et al. (2012); Sedighi } \text { et al. (2015); Nouri } \\
\text { et al. (2013) }\end{array}$ \\
\hline 5 & Investimentos na implementação & $\begin{array}{l}\text { Wickramasinghe } \text { et al. (2007); Du Plessis (2008; } \\
\text { Valmohammadi (2010); Sedighi et al. (2015); Sedighi et al. } \\
\text { (2015); Oliveira et al. (2012) }\end{array}$ \\
\hline 7 & $\begin{array}{l}\text { Estrutura organizacional } \\
\text { alinhada com GC }\end{array}$ & $\begin{array}{l}\text { Du Pleassis (2008); Valmohammadi (2010); Mirpoor et al. } \\
\text { (2013); Sedighi } \text { et al. (2015); Oliveira et al. (2012); Wang e } \\
\text { Chang (2007); }\end{array}$ \\
\hline Falta de incentivo & $\begin{array}{l}\text { Lin e Tseng (2005); Siddique (2012); Du Plessis (2008); } \\
\text { Anand, Kant e Singh (2013) }\end{array}$ \\
\hline
\end{tabular}

Fonte: Os autores (2018)

Perspectivas em Gestão \& Conhecimento, João Pessoa, v. 8, n. 2, p. 43-61, mai./ago. 2018. 
Observando os estudos apresentados, verificou-se que é possível uma complementaridade nas três maneiras de abordagem sobre barreiras. As barreiras podem ser identificadas de maneira geral, relacionando todas que possam interferir em uma implantação genérica. Mas a identificação em países, tipo de empresas ou setores específicos pode proporcionar uma maior precisão, enfocando aquelas que, de fato, interessam em determinados tipos de organizações.

A partir da identificação é possível a hierarquização das barreiras, definindo quais devem ser trabalhadas numa primeira ação, o que pode facilitar a gestão do processo de implementação, e eventualmente reduzir custos e tornar as ações efetivas. Aliada a análise em tipos ou setores específicos, acentua-se o foco das ações. A classificação das barreiras em uma organização específica também é apresentada, embora seja um único caso localizado.

As discussões que procuram analisar em profundidade uma barreira em especial, discutindo possíveis soluções ou ainda possibilitando uma previsão de sucesso, complementam o debate anterior e abrem possibilidades para avançar ainda mais nesse tema.

Mas independentemente da abordagem realizada, o que se percebe é que todos os trabalhos se preocupam com uma implementação de GC de forma efetiva nas organizações, pois de nada serve apenas ter algum sistema de GC, sendo necessário que esse realmente auxilie a organização a atingir seus objetivos.

\section{CONSIDERAÇÕES FINAIS}

A implementação de $\mathrm{GC}$ exige um investimento substancial e altera a cultura e a estrutura da organização. Muitos fatores afetam a implementação, e entender como esses elementos afetam um programa de GC é importante para construir mecanismos de superação. A literatura científica referencia-se a estes elementos como dificultadores, limitadores, fatores críticos ou barreiras.

Uma contribuição desse estudo é a proposição de classificação da literatura científica sobre barreiras na implementação de GC em três abordagens diferentes: identificando barreiras, classificando-as por grau de importância ou realizando uma discussão sobre as mesmas. Além dessa classificação macro, os trabalhos que objetivam identificar as barreiras ainda podem ser subdivididos entre três abordagens: identificando de maneira geral, em um país, setor ou tipo de organização ou ainda as que procuram identificar em empresas específicas. Os trabalhos que hierarquizam também se dividem em os que classificam de maneira geral, de maneira específica em um país, setor ou tipo de organização ou classificam em organizações específicas, podendo criar métodos para isso. Já os que discutem sobre as barreiras tratam de temas específicos, discutem soluções ou realizam previsões.

Outra contribuição é a identificação das principais barreiras apresentadas na literatura que foram, em ordem de importância: cultura organizacional não alinhada; falta de engajamento e apoio da liderança; dificuldades com sistemas/tecnologia; investimentos na implementação; estrutura organizacional; má compreensão da GC em teoria e prática; estratégia organizacional não alinhada com GC; e falta de incentivo.

Esta pesquisa limitou-se às barreiras relacionadas estritamente à implementação de GC nas organizações. Dessa forma, existem outras barreiras, limitadores ou dificultadores para os processos de gestão do conhecimento, mas não fizeram parte do escopo desta pesquisa. Outra limitação está no não aprofundamento de cada barreira, que pode esclarecer quais atividades ou estratégias podem ser utilizadas para driblar e obter êxito na implementação.

Como sugestão de trabalhos futuros recomenda-se o estudo de múltiplas análises empíricas para comprovar as barreiras listadas nessa pesquisa. Esses estudos de casos, sejam qualitativos ou quantitativos, podem colaborar para uma melhor compreensão dos impactos

Perspectivas em Gestão \& Conhecimento, João Pessoa, v. 8, n. 2, p. 43-61, mai./ago. 2018. 
de cada um dos elementos dificultadores, barreiras ou fatores críticos que possam a comprometer uma boa implementação da GC.

\section{REFERÊNCIAS}

AHMAD, H. S.; AN, M. Knowledge management implementation in construction projects: a KM model for Knowledge Creation, Collection and Updating (KCCU). International Journal of Project Organisation and Management, v. 1, n. 2, p. 133-166, 2008.

ANAND, A.; KANT, R.; SINGH, M. D. Knowledge sharing in SMEs: Modelling the barriers. International Journal of Management and Enterprise Development, v. 12, n. 4-6, p. 385-410, 2013.

ANAND, A., KANT, R., PATEL, D. P.; SINGH, M. D. Knowledge management implementation: a predictive model using an analytical hierarchical process. Journal of the Knowledge Economy, 6(1), 48-71, 2015.

BARDIN, L. Análise de conteúdo. São Paulo: Edições 70, 2011.

CEN - European guide to good practice in knowledge management. CEN Workshop Agreement CWA14924-3. 2004.

DALKIR, K. L.. Knowledge management in theory and practice. Boston: Elsevier, 2005.

DU PLESSIS, M. What bars organisations from managing knowledge successfully? International Journal of Information Management, v. 28, n. 4, p. 285-292, 2008.

ELSEVIER. Disponível em: http://www.americalatina.elsevier.com/sul/pt-br/scopus.php. Acesso em: 06 set. 2016.

FINESTONE, N.; SNYMAN, R. Corporate South Africa: Making multicultural knowledge sharing work. Journal of Knowledge Management, v. 9, n. 3, p. 128-141, 2005.

GOLD, A. H.; MALHOTRA A., SEGARS, A. H. Knowledge management: An organizational capabilities perspective. Journal of management Information Systems, v. 18, n. 1, p. 185-214, 2001.

GOMES JR, W. V. Gestão do conhecimento e mapeamento de competências: um estudo de caso. 2013. 231 fls. Dissertação (Mestrado em Engenharia e Gestão do Conhecimento) Programa de Pós-Graduação em Engenharia e Gestão do Conhecimento, UFSC. Florianópolis, 2013.

GREENHALGH, T. Papers that summarise other papers (systematic reviews and meta-analyses). BMJ: British Medical Journal, v. 315, n. 7109, p. 672, 1997.

KANT, R.; SINGH, M. D. Knowledge management implementation: Modeling the barriers. Journal of Information and Knowledge Management, v. 7, n. 4, p. 291-305, 2008.

Perspectivas em Gestão \& Conhecimento, João Pessoa, v. 8, n. 2, p. 43-61, mai./ago. 2018. 
Knowledge management implementation: Modelling the variables. International Journal of Innovation and Learning, v. 6, n. 3, p. 342-361, 2009.

KHALIFA, Z. A.; JAMALUDDIN, Y. Key success factors affecting knowledge management implementation in construction industry in Libya. Australian Journal of Basic and Applied Sciences, v. 6, n. 5, p. 161-164, 2012.

LIN, C.; TSENG, S. M. The implementation gaps for the knowledge management system. Industrial Management and Data Systems, v. 105, n. 2, p. 208-222, 2005.

MACEDO, M.; FIALHO, F.; MITIDIERI, T. C.; SANTOS, N. Gestão do conhecimento organizacional. Florianópolis: Editora da UFSC, 2010. 200 p.

MILOSZ, M.; MILOSZ, E. Critical success factors and barriers to implementation of knowledge management systems at Polish SMEs. Actual Problems of Economics, n. 6, p. 309-315, 2010.

NAIR, Praba; PRAKASH, Kamlesh (eds.) Knowledge management: facilitator's guide. APO: Tokyo, 2009.

NOURI, R.; MOSHABAKI, A.; RAISSI, S.; JAVADINIA, Y. The influence of the personalization and codification strategies on successful knowledge management case study: National Iranian oil company. Research Journal of Applied Sciences, Engineering and Technology, v. 6, n. 6, p. 989-996, 2013.

OLIVEIRA, M.; CALDEIRA, M.; BATISTA ROMÃO, M. J. Knowledge Management Implementation: An Evolutionary Process in Organizations. Knowledge and Process Management, v. 19, n. 1, p. 17-26, 2012.

PICKER, S.; RUHNKE, A.; LEKER, J. Developing knowledge management -what makes the success? International Journal of Technology Management, v. 45, n. 3-4, p. 380-389, 2009.

MIRPOOR, S. S.; HAGHIGH, R. R.; ANSARI, A.; AHMADI, T. A.. The study and comparison of the effective factors on implementation of knowledge management case study: Customs supervision office of Bushehr Province. Advances in Environmental Biology, v. 7, n. 10, p. 3116-3123, 2013.

SALDAÑA, J. An introduction to codes and coding. The coding manual for qualitative researchers, Los Angeles: Sage, 2009.

SEDIGHI, M.; VAN SPLUNTER, S.; ZAND, F.; BRAZIER, F . Evaluating Critical Success Factors model of knowledge management: An Analytic hierarchy process (AHP) approach. International Journal of Knowledge Management, v. 11, n. 3, p. 17-36, 2015.

SERVIN, G. ABC of knowledge management. NHS National Library for Health, 2005.

SHANG, S. The method of selecting critical successful factors to knowledge management and its automation. Journal of Theoretical and Applied Information Technology, v. 49, n. 1, p. 433441, 2013.

Perspectivas em Gestão \& Conhecimento, João Pessoa, v. 8, n. 2, p. 43-61, mai./ago. 2018. 
SHOO, R.; MATUKU, W.; IRERI, J.; NYAGERO, J.; GATONGA, P. The place of knowledge management in influencing lasting health change in Africa: an analysis of AMREF's progress. The Pan African medical journal, v. 13 Suppl 1, p. 3, 2012.

SIDDIQUE, C. M. Knowledge management initiatives in the United Arab Emirates: A baseline study. Journal of Knowledge Management, v. 16, n. 5, p. 702-723, 2012.

VALMOHAMMADI, C. Investigation and assessment of critical success factors of knowledge management implementation in Iranian small-to-medium sized enterprises. Journal of Applied Sciences, v. 10, n. 19, p. 2290-2296, 2010.

WANG, T. C.; CHANG, T. H. Application of consistent fuzzy preference relations in predicting the success of knowledge management implementation. European Journal of Operational Research, v. 182, n. 3, p. 1313-1329, 2007.

WICKRAMASINGHE, N.; BALI, R. K.; GEISLER, E. The major barriers and facilitators for the adoption and implementation of knowledge management in healthcare operations. International Journal of Electronic Healthcare, v. 3, n. 3, p. 367-381, 2007.

WIIG, K. M. Knowledge management: an introduction and perspective. Journal of Knowledge Management, v. 1, n. 1, p. 6-14, 1997

WU, W. W. Segmenting critical factors for successful knowledge management implementation using the fuzzy DEMATEL method. Applied Soft Computing Journal, v. 12, n. 1, p. 527-535, 2012.

Artigo recebido em 03/03/2017 e aceito para publicação em 26/03/2018 